\title{
Wavelength- and Time-Selective Reconfigurable Optical Add/Drop Multiplexer Using Time-Frequency Domain Processing
}

\author{
Tsuyoshi Konishi, Ryosuke Itoh, and Kazuyoshi Itoh \\ Graduate School of Engineering, Osaka University, 2-1 Yamadaoka, Suita, Osaka 565-0871, Japan \\ Correspondence should be addressed to Tsuyoshi Konishi, konishi@mls.eng.osaka-u.ac.jp
}

Received 26 December 2009; Accepted 19 March 2010

Academic Editor: Syed Ismail Shah

Copyright ( 2010 Tsuyoshi Konishi et al. This is an open access article distributed under the Creative Commons Attribution License, which permits unrestricted use, distribution, and reproduction in any medium, provided the original work is properly cited.

We propose and demonstrate a wavelength- and time-selective reconfigurable optical add/drop multiplexer (ROADM) using timefrequency domain processing. The proposed ROADM is realized by allocating wavelength channels and time slots to corresponding 2D spatial channels on a MEMS optical switch. Experimental results show the wavelength- and time-selective drop operation for a signal with equivalent $3.2 \mathrm{~Tb} / \mathrm{s}(0.64 \mathrm{~Tb} / \mathrm{s} \times 5$ channels $)$, and the reconfigurability by the switching operation of the MEMS optical switch.

\section{Introduction}

Reconfigurable optical add/drop multiplexers (ROADMs) are the key elements for building the next-generation dynamically reconfigurable optical networks [1]. ROADMs are required for the add/drop and cut-through of individual or multiple wavelength-division multiplexed channels and time-division multiplexed slots at the network nodes $[1,2]$. Besides, the combination of wavelength-division multiplexing (WDM) and optical time-division multiplexing (OTDM) technologies has become the fundamental scheme of the recent transmission system which allows the total transmission capacity of over $20 \mathrm{~Tb} / \mathrm{s}$ [3]. Therefore, to achieve more flexible and scalable networks, ROADMs should have add/drop and cut-through functions for not only wavelength channels but also time slots. Since such a simultaneous treatment of 2-D multiplexed signals employing OTDM and WDM technologies (OTDM/WDM signals) is equivalent to time-frequency domain processing, ROADMs for OTDM/WDM signals should be developed based on it. Up to now, the innovative proposals of time-selective ROADMs are very few despite their needs $[2,4]$. In contrast, there have been various proposals on wavelength-selective ROADMs [5]. Especially, diffraction-grating-based free-space dispersive optics coupled to a microelectromechanical systems (MEMS) optical switch are most mature technologies [6]. An optical switch based on free-space optics plays an important role in such ROADMs with respect to stability, scalability, compactness, and low insertion loss. In addition, recently, a two-dimensional (2-D) spatial switching device, which is a promising configuration for further integration, has been actively developed [7]. OTDM/WDM signals can be treated as independent multiple-bits signals in the time-frequency domain (2-D space as mathematical picture) $[8,9]$. In this sense, it is natural that each demultiplexed unit bit in an OTDM/WDM signal can be fed on a 2-D spatial switching device for individual processing. For the realization of a wavelength- and time-selective ROADM with the full advantages of 2-D spatial switching devices, 2-D time-space conversion techniques [10-13] are inevitably indispensable for spatial allocation of wavelength channels and time slots. Since this 2-D time-space conversion technique is based on time-frequency transform, it is expected to utilize unique features derived from time-frequency domain processing too.

In this paper, we propose a wavelength- and timeselective ROADM using 2-D time-space conversion and a MEMS optical switch based on time-frequency domain processing. We experimentally demonstrate the drop and cut-through operations for the proposed ROADM because an add function of the proposed ROADM can be easily achieved. 


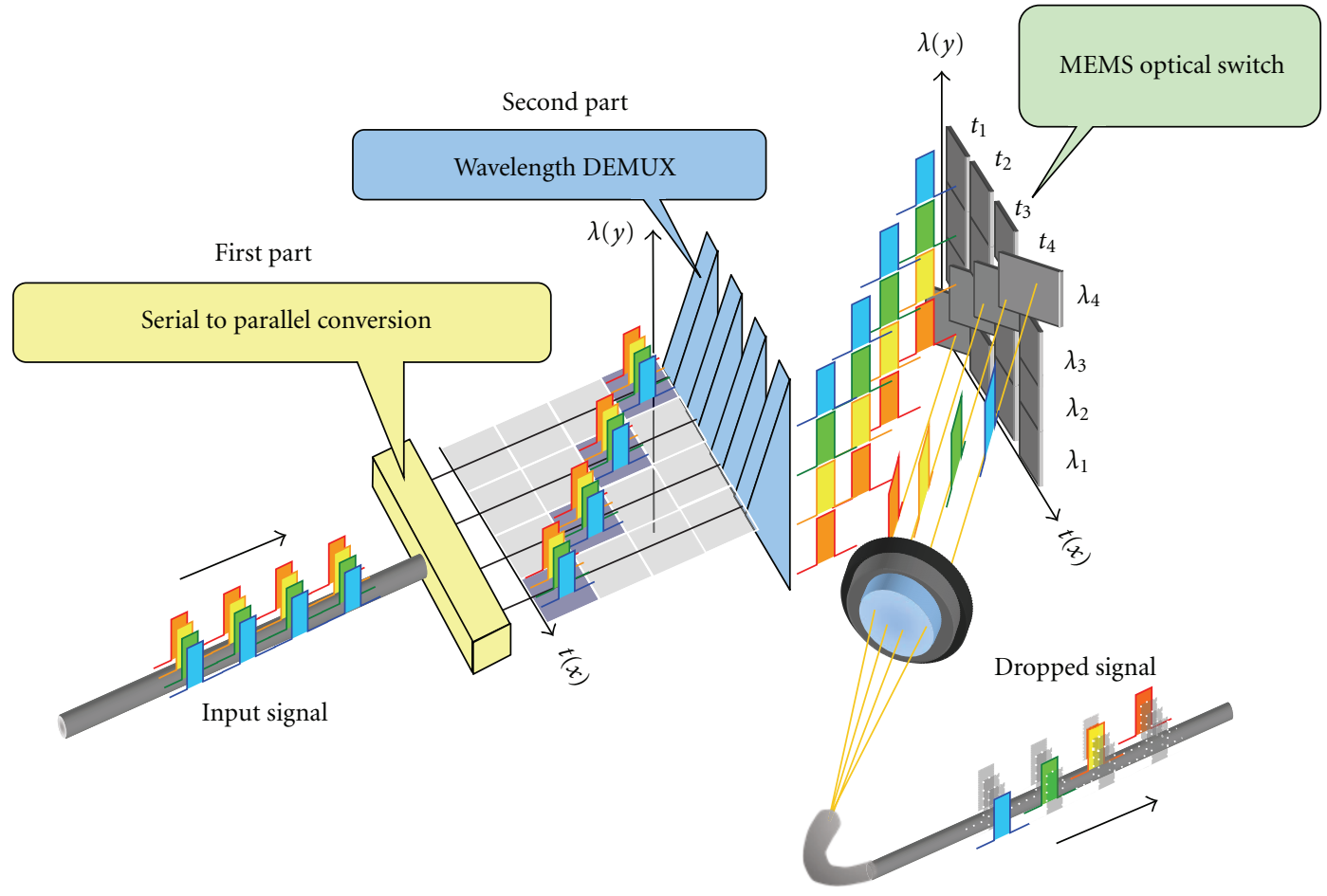

FIGURE 1: The schematic diagram of the proposed wavelength- and time-selective reconfigurable optical add/drop multiplexer.

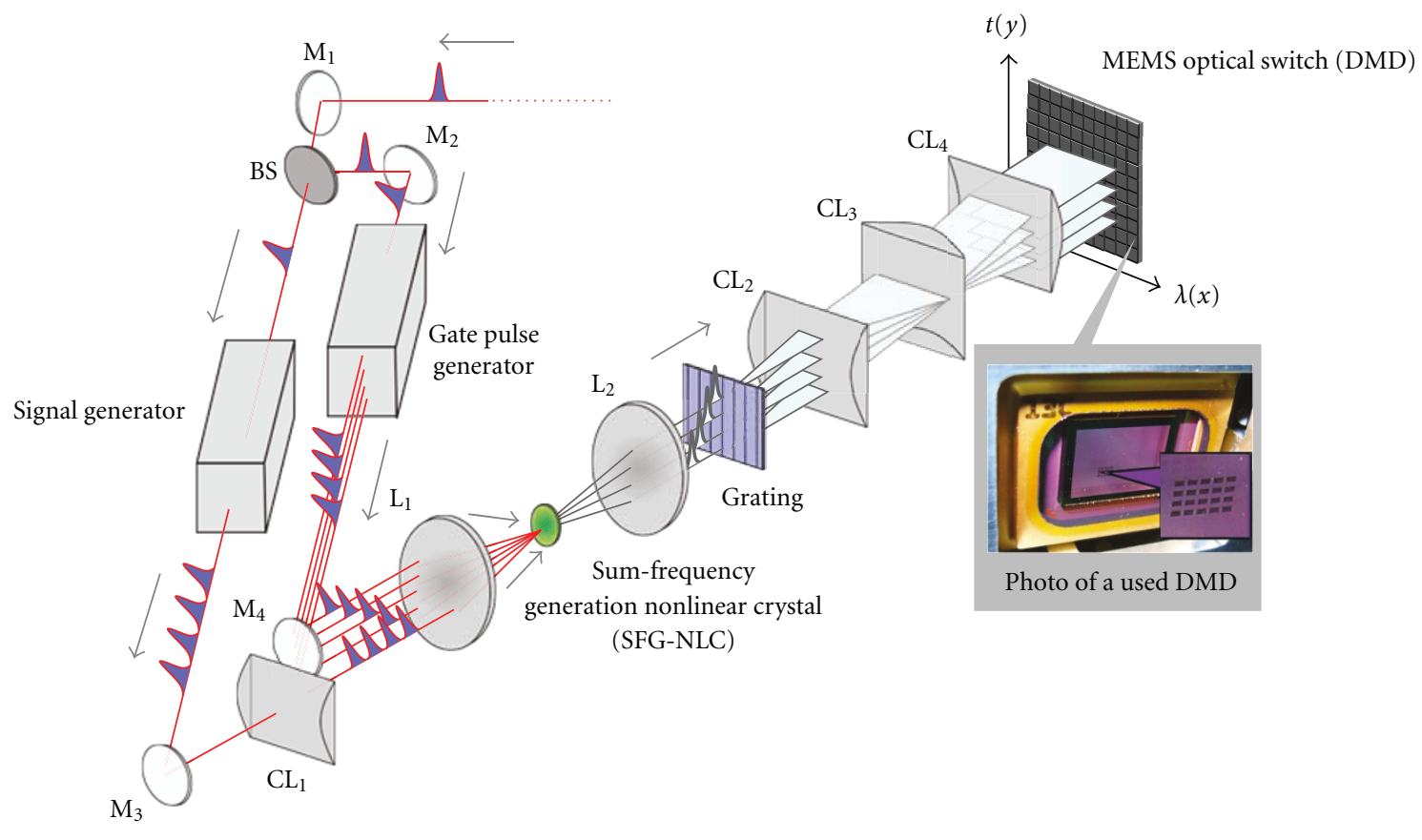

M: Mirror

L: Lens

BS: Beam splitter

CL: Cylindrical lens

Figure 2: Experimental setup for the proposed ROADM. 


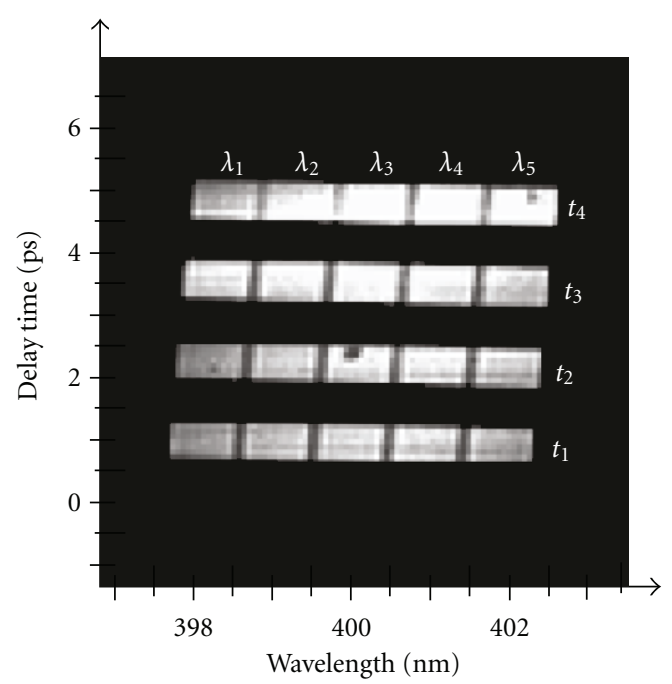

FIgUre 3: The spatial distribution of whole wavelength channel and time slot in fit a state for switching by MEMS optical switch.

\section{Principle and Experimental Setup}

The schematic diagram of the proposed wavelength- and time-selective ROADM is shown in Figure 1. 2-D time-space conversion is composed of two parts. The first part is a serial-to-parallel conversion for demultiplexing time slots. A wavelength- and time-division multiplexed signal is fed to a serial-to-parallel conversion section. Then, each time slot in an input signal is spatially allocated along the $X$-axis. Next, each time slot, which is spatially divided, is fed to a wavelength demultiplexing section. Then, each wavelength channel is spatially allocated along the $Y$-axis. Consequently, each time slot and wavelength channel is allocated to a corresponding to spatial channel on the single 2-D spatial plane. A MEMS optical switch is placed on that plane to switch time slots and wavelength channels. For example, time slots and wavelength channels corresponding spatial channels can be simultaneously dropped by switching spatial channels. And, we can change the dropped wavelength channels and time slots by changing the dropped spatial channels.

Here, we focus on verification of drop and cutthrough operations and the reconfigurability of the proposed ROADM because insertion of wavelength channels and time slots for add operation can be also achieved by setting up almost the same optical system for drop and cut-through operations.

Our experimental setup for the proposed ROADM is shown in Figure 2. In this experiment, we used an ultrashort pulse radiated from a mode-locked Ti:sapphire laser (Millenia pro pumped Tsunami System; Spectra Physics, Inc.) as a light source. The pulse width, the center wavelength, and the repetition rate were $100 \mathrm{fs}, 800 \mathrm{~nm}$, and $82 \mathrm{MHz}$, respectively. We used a four-bit ultrashort pulse sequence of a 1.56 ps interval in which 5 wavelength channels and 4 time slots are multiplexed (an input signal). To realize a serial-to-parallel conversion in this experiment, we used a sum-frequency generation nonlinear crystal (SFG-NLC) and a four-bit ultrashort pulse sequence as a time-gating device and a gate pulse sequence, respectively. Because an SFG-NLC was used as a time-gating device in this experiment, the low light efficiency of SFG, which is inherently not very high, mainly affects the light efficiency of the entire system. To cover the low light efficiency, we used a light source in a near-infrared band that provided higher power than that of a communication band. As an SFG-NLC, we used a $B-\mathrm{BaB}_{2} \mathrm{O}_{4}$ (B.B.O) NLC (CASIX, Inc.). The crystal cut and orientation were designed to satisfy the type I phase-matching condition for noncollinear sum-frequency generation. As shown in Figure 2, we used a B.B.O, a transmission deep grating (DG) of 2500 lines/mm, lenses $\left(\mathrm{L}_{1}, \mathrm{~L}_{2}\right)$ with focal length of 100 , $40 \mathrm{~mm}$, respectively, cylindrical lenses $\left(\mathrm{CL}_{1}, \mathrm{CL}_{2}, \mathrm{CL}_{3}, \mathrm{CL}_{4}\right)$ with focal length of $100,100,200,100 \mathrm{~mm}$, respectively, a beam splitter (BS), mirrors $\left(\mathrm{M}_{1}, \mathrm{M}_{2}, \mathrm{M}_{3}, \mathrm{M}_{4}\right)$, and a charge-coupled device (CCD) camera. And, we used a digital micromirror device (DMD Discovery 1100; Productivity Systems, Inc.) as a MEMS optical switch. An ultrashort pulse was split into two beams by a beam splitter. The first was fed to a signal generator composed of two Michelson interferometers for the application of artificial wavelength- and time-division multiplexed signal; the second was fed to a gate pulse generator composed of two Michelson interferometers and four rectangular prisms for the generation of a gate pulse sequence. A signal pulse sequence and a gate pulse sequence that had spatially different heights were incident into an SFGNLC by a lens $\mathrm{L}_{1}$. Only when two pulses were simultaneously incident into an SFG-NLC, an SFG pulse was generated. Each gate pulse was incident into an SFG-NLC at a different angle; therefore, each SFG pulse was output to a different direction under a phase-matching condition with keeping a time delay corresponding to a time slot interval. That is, an SFG-NLC worked as a time-gating device. Each SFG pulse that had a different output direction was collimated by a lens $\mathrm{L}_{2}$, then, each time slot was allocated to a corresponding spatial channel along the vertical axis. And, we used a transmission DG [14] to obtain high diffraction efficiency for a wavelength demultiplexing. After a serial-to-parallel conversion, each SFG pulse was dispersed into decomposed spectral components using a transmission DG and cylindrical lens $\mathrm{CL}_{3}$, then, each wavelength channel was allocated to a corresponding spatial channel along the horizontal axis. Consequently, spatial channels along the horizontal and vertical axes on the single 2-D spatial plane correspond to wavelength channels and time slots, respectively. DMD as a MEMS optical switch was placed on that plane to drop spatial channels. In this experiment, we measured spatial channels by a CCD camera and the temporal waveform and the spectrum of the dropped signal by a cross-correlation method and a spectrometer, respectively.

\section{Experimental Results and Discussion}

Experimental results are shown in Figures 3, 4, 5, and 6. Figure 3 shows that whole wavelength channel and time slot in a signal pulse sequence are allocated to corresponding 


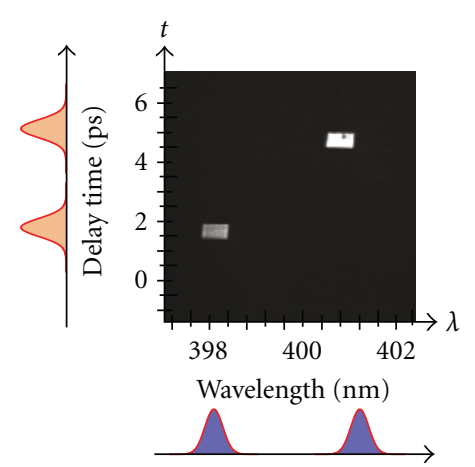

(a)

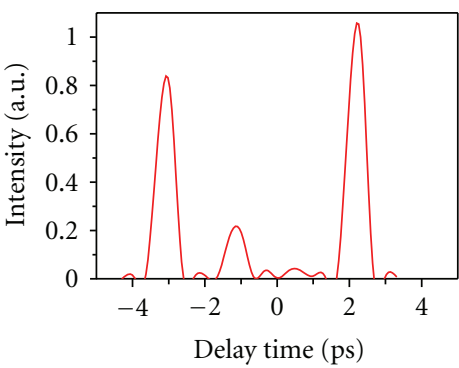

(b)

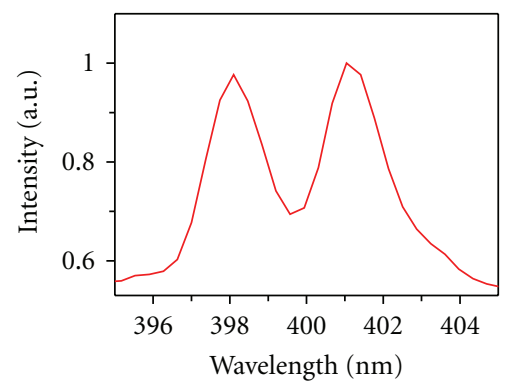

(c)

FIgURE 4: Experimental results for dropped signal $\left(\lambda_{1}, t_{1}\right)$ and (b) $\left(\lambda_{5}, t_{4}\right)$ : (a) spatial distribution, (b) temporal waveform, and (c) spectrum.

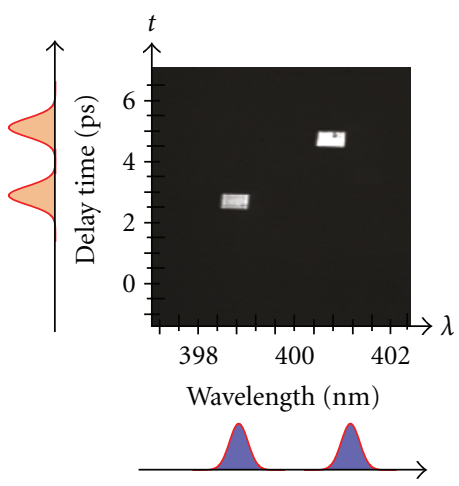

(a)

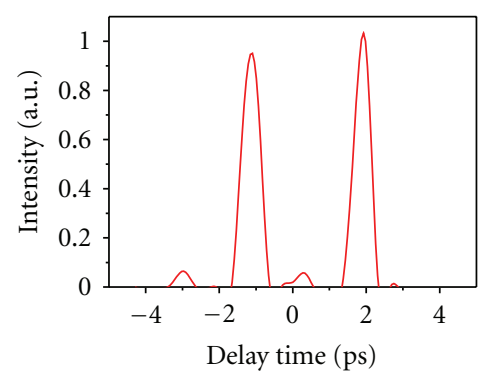

(b)

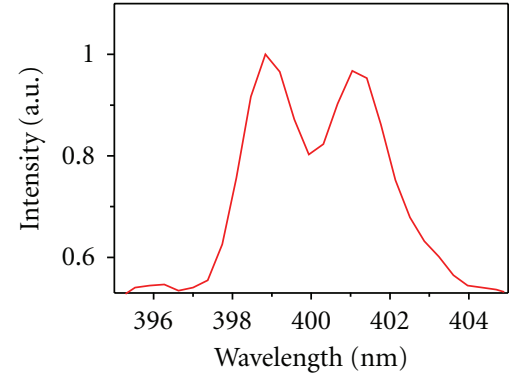

(c)

Figure 5: Experimental results for dropped signal $\left(\lambda_{1}, t_{1}\right)$ and (b) $\left(\lambda_{5}, t_{4}\right)$ after reconfigure operation: (a) spatial distribution, (b) temporal waveform, and (c) spectrum.

spatial channels along the horizontal and vertical axes on the MEMS optical switch by 2-D time-space conversion. From this result, we could verify that whole wavelength channels and time slots of OTDM/WDM signals are successfully demultiplexed on the single spatial plane for drop and cut-through operations. Spatial distributions on the MEMS optical switch are divided into a $(5 \times 4)$ spatial channel matrix. We define each spatial channel as $\left(\lambda_{m}, t_{n}\right)$.

Next, to verify drop and cut-through operations of the used MEMS optical switch, we focused on two spatial channels. Figure 4(a) shows the spatial distribution of selected spatial channels for drop or cut-through operations. Spatial channels are corresponding to $\left(\lambda_{1}, t_{1}\right)$ and $\left(\lambda_{5}, t_{4}\right)$ in Figure 4(a). To verify the wavelength- and time-selective drop and cut-through operation, we measured the temporal waveform and the spectrum of dropped channels which are corresponding to $\left(\lambda_{1}, t_{1}\right)$ and $\left(\lambda_{5}, t_{4}\right)$. The temporal waveform and the spectrum of $\left(\lambda_{1}, t_{1}\right)$ and $\left(\lambda_{5}, t_{4}\right)$ are described in Figures 4(b) and 4(c), respectively. From these results, we could confirm the wavelength- and time-selective drop and cut-through operations of the proposed ROADM. In the experiment for drop and cut-through operations, we used 5 wavelength channels in a four-bit ultrashort pulse sequence of a $1.56 \mathrm{ps}$ interval as an input signal. The operation speed of drop and cut-through operations can be estimated to equivalent $3.2 \mathrm{~Tb} / \mathrm{s}(0.64 \mathrm{~Tb} / \mathrm{s} \times 5$ channels $)$ capacity because these operations themselves do not need reconfigure operation.

To verify the reconfigure operation, we changed the dropped wavelength channels and time slots by changing switching condition of a MEMS optical switch. The spatial distribution, temporal waveform, and the spectrum of $\left(\lambda_{2}\right.$, $\left.t_{2}\right)$ and $\left(\lambda_{5}, t_{4}\right)$ are shown in Figures 5(a), 5(b), and $5(\mathrm{c})$, respectively. From these results, we could confirm reconfigure operation of the proposed ROADM compared with the result in Figure 4.

Finally, we measured response of reconfigure operation by the MEMS optical switch used. To measure the response speed for reconfigure operation, $\left(\lambda_{3}, t_{1}\right)$ was coupled into the MEMS optical switch. The reconfigured signals of onstate and off-state were directly detected by two PINphotodetectors (ET-4000, ET-2030; Electro-Optics Technology, Inc.) and captured by an oscilloscope (Infinium; Agilent $(\mathrm{HP}))$, respectively. The measurement results are shown in Figure 6. Ch. 1 and ch. 2 were corresponding to the dropped signal and the cut-through signal, respectively. Here, we set on-state signal of $\left(\lambda_{3}, t_{1}\right)$ as the dropped signal and the off-state signal of $\left(\lambda_{3}, t_{1}\right)$ as the cut-through signal. The 


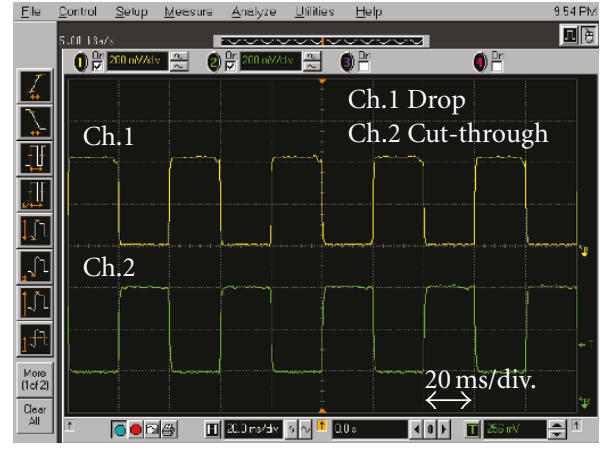

(a)

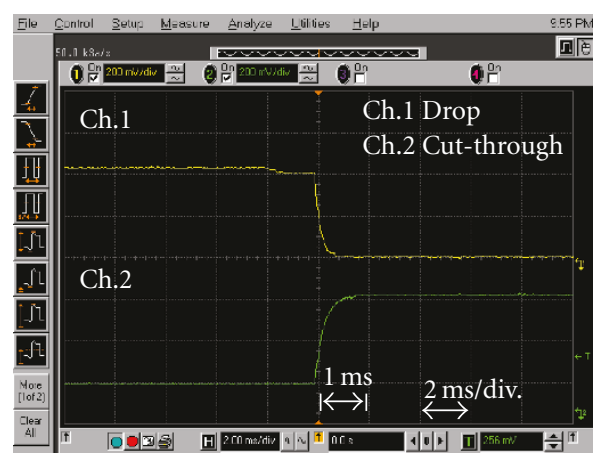

(b)

FIGURE 6: Measured response of reconfigure operation by the MEMS optical switch: (a) $20 \mathrm{~ms} / \mathrm{div}$ and (b) $2 \mathrm{~ms} / \mathrm{div}$.

horizontal scales of Figures 6(a) and 6(b) were $20 \mathrm{~ms} / \mathrm{div}$. and $2 \mathrm{~ms} /$ div. From these results, the operation speed of reconfigure operation by a MEMS optical switch could be estimated to be about $1 \mathrm{~ms}$.

In these experiments, we verified the operation of the proposed ROADM using a light source in a near-infrared band. The operation in an optical communication band will demand a few changes of devices such as a time-gating device, lens, and grating. Among the most important is a time-gating device. In the proposed ROADM, we utilized an SFG process in a B.B.O crystal as a time gate for a serial-toparallel conversion. A B.B.O crystal has relatively a low SFG efficiency in an optical communication band. In addition, an SFG process inherently causes a wavelength conversion of an input signal. If we adopt the proposed ROADM to actual optical networks, we should utilize an SFG-NLC which has high SFG efficiency in an optical communication band, and the converted wavelength should be turned back to an available one. As the promising devices to fill those needs, a 2-adamantylamino-5-nitropyridine (AANP) as a high SFG efficiency device has been reported [15]. And, a cascaded SFG and difference-frequency-generation(DFG-) based highly-efficient wavelength conversion in a periodically poled $\mathrm{LiNbO}_{3}$ (PPLN) has been also reported [16]. These devices can be applied to the proposed ROADM.

\section{Conclusion}

We experimentally demonstrated drop and cut-through operations for a $0.64 \mathrm{~Tb} / \mathrm{s} \times 5$ channels OTDM/WDM signal with the proposed wavelength- and time-selective ROADM. In addition, the reconfigure operation of switching condition by a MEMS optical switch shows about $1 \mathrm{~ms}$ switching response. To adopt the proposed ROADM to actual optical networks, the previously reported highly-efficient timegating device and the wavelength converter can be applied to our proposed ROADM. Our proposed ROADM can introduce a bitwise adaptive signal compensation technique which is one of the unique techniques derived from timefrequency domain processing too [17]. In addition, our proposed ROADM could be used for a pulse synthesizer to investigate a photoresponse of biomaterials in the field of laser spectroscopy.

\section{References}

[1] R. Shankar, M. Florjańczyk, T. J. Hall, A. Vukovic, and H. Hua, "Multi-degree ROADM based on wavelength selective switches: architectures and scalability," Optics Communications, vol. 279, no. 1, pp. 94-100, 2007.

[2] A. T. Clausen, L. Oxenlowe, A. Siahlo, J. Seoane, and P. Jeppesen, "Expansion of point-to-point OTDM systems to a ring network," in Proceedings of European Conference on Optical Communication (ECOC '04), 2004, Th2.6.3.

[3] H. Masuda, A. Sano, T. Kobayashi, et al., "20.4-Tb/s $(204 \times 111 \mathrm{~Gb} / \mathrm{s})$ Transmission over $240 \mathrm{~km}$ using BandwidthMaximized Hybrid Raman/EDFAs," in Proceedings of the Optical Fiber Communication Conference (OFC '07), 2007, PDP20.

[4] J. Seoane, A. T. Clausen, L. K. Oxenløwe, M. Galili, T. Tokle, and P. Jeppesen, "Enabling technologies for OTDM networks at $160 \mathrm{Gbit} / \mathrm{s}$ and beyond," in Proceedings of the 18th Annual Meeting of the IEEE Lasers and Electro-Optics Society (LEOS '05), pp. 81-82, Sydney, Australia, October 2005, MG1.

[5] B. P. Keyworth, "ROADM subsystems \& technologies," in Proceedings of Optical Fiber Communication Conference (OFC/NFOEC '05), vol. 3, pp. 45-48, Anaheim, Calif, USA, March 2005, OWB5.

[6] S. A. Khan and N. A. Riza, "Demonstration of the MEMS digital micromirror device-based broadband reconfigurable optical add-drop filter for dense wavelength-division-multiplexing systems," Journal of Lightwave Technology, vol. 25, no. 2, pp. 520-526, 2007.

[7] M. Yano, F. Yamagishi, and T. Tsuda, "Optical MEMS for photonic switching-compact and stable optical crossconnect switches for simple, fast, and flexible wavelength applications in recent photonic networks," IEEE Journal on Selected Topics in Quantum Electronics, vol. 11, no. 2, pp. 383-394, 2005.

[8] L. Cohen, “Time-frequency distributions-a review," Proceedings of the IEEE, vol. 77, no. 7, pp. 941-981, 1989.

[9] I. Shafi, J. Ahmad, S. I. Shah, and F. M. Kashif, "Techniques to obtain good resolution and concentrated time-frequency distributions: a review," EURASIP Journal on Advances in Signal Processing, vol. 2009, Article ID 673539, 43 pages, 2009. 
[10] T. Konishi and Y. Ichioka, "Ultrafast image transmission by optical time-to-two-dimensional-space-to-time-to-twodimensional-space conversion," Journal of the Optical Society of America A, vol. 16, no. 5, pp. 1076-1088, 1999.

[11] Y. Oshita, T. Konishi, and Y. Ichioka, "Ultrafast time-totwo-dimensional-space conversion system using SHG crystal," Optical Review, vol. 9, no. 4, pp. 141-145, 2002.

[12] Y. Oshita, T. Konishi, W. Yu, K. Itoh, and Y. Ichioka, "Application of ultrafast time-to-two-dimensional-space-totime conversion (II): time-varying spectral control for arbitrary ultrafast signal reshaping," IEEE Photonics Technology Letters, vol. 16, no. 2, pp. 623-625, 2004.

[13] T. Konishi, Y. Oshita, W. Yu, H. Furukawa, K. Itoh, and Y. Ichioka, "Application of ultrafast time-to-two-dimensionalspace-to-time conversion (I): time-varying spectral modulation for arbitrary ultrafast signal generation," IEEE Photonics Technology Letters, vol. 16, no. 2, pp. 620-622, 2004.

[14] W. Yu, Y. Oshita, H. Toyota, T. Konishi, T. Yotsuya, and Y. Ichioka, "Development of deep gratings for wavelength dispersion applications," Japanese Journal of Applied Physics, vol. 42, no. 6, pp. 3434-3437, 2003.

[15] S. Tomaru, S. Matsumoto, T. Kurihara, H. Suzuki, N. Ooba, and T. Kaino, "Nonlinear optical properties of 2adamantylamino-5-nitropyridine crystals," Applied Physics Letters, vol. 58, no. 23, pp. 2583-2585, 1991.

[16] C.-Q. Xu and B. Chen, "Cascaded wavelength conversions based on sum-frequency generation and difference-frequency generation," Optics Letters, vol. 29, no. 3, pp. 292-294, 2004.

[17] T. Konishi, Y. Oshita, and K. Itoh, "Ultrafast optical distortion equalizer using time-frequency domain processing," Journal of Lightwave Technology, vol. 24, no. 7, pp. 2693-2700, 2006. 